\title{
Anomalous bias factors of dislocations in bcc iron
}

\author{
Zhongwen Chang ${ }^{\mathrm{a}}$, Dmitry Terentyev ${ }^{\mathrm{b}}$, Nils Sandberga,c $^{\mathrm{a}, \mathrm{c}}$ Karl Samuelsson ${ }^{\mathrm{a}}$, \\ Pär Olsson ${ }^{\mathrm{a}}$ \\ ${ }^{a}$ KTH Royal Institute of Technology, Reactor Physics, Roslagstullsbacken 21, SE-10691 \\ Stockholm, Sweden \\ ${ }^{b} S C K-C E N$, Nuclear Materials Science Institute, Boeretang 200, B-2400 Mol, Belgium \\ ${ }^{c}$ Swedish Radiation Safety Authority, Solna Strandväg 96, SE-171 16 Stockholm, Sweden
}

\section{Abstract}

Dislocation bias factors in bcc Fe have been calculated based on atomistic interaction energy maps on three kinds of dislocations, namely the $a_{0} / 2\langle 111\rangle\{110\}$ screw, $a_{0} / 2\langle 111\rangle\{110\}$ and $a_{0}\langle 100\rangle\{001\}$ edge dislocations. The results show that the dislocation bias is higher for the $a_{0} / 2\langle 111\rangle$ edge dislocation than for the $a_{0}\langle 100\rangle$ edge dislocation, even though the latter possesses a larger Burgers vector. This indicates the importance of the dislocation core contribution. For the $a_{0} / 2\langle 111\rangle\{110\}$ screw dislocation, a negative dislocation bias has been obtained, which implies a more efficient absorption of vacancies than of SIAs. The effect of coexistence of both edge- and screw dislocations are assessed by a total bias. A possible complementary mechanism for explaining the long swelling incubation time in bcc metals is suggested and discussed.

Keywords: Dislocation bias, Atomistic calculation, Interaction energy, bcc

Email addresses: zhongwen@kth.se (Zhongwen Chang), dterenty@sckcen.be (Dmitry Terentyev), polsson@kth.se (Pär Olsson) 


\section{Introduction}

Classical radiation damage theory underlines the importance of the dislocation bias, which physically is the preference of absorption of self-interstitial atoms (SIAs) over vacancies by dislocation lines [1]. Such a fundamental radiation effect as void swelling was predicted in 1959 by Greenwood, Foreman and Rimmer (GFR) on the basis of the assumption that radiation damage produces 3-D migrating point defects, SIAs and vacancies, which cluster and contribute to the growth of the dislocation density and of the voids. However, void growth is established due to the growing excess of vacancies in the bulk because SIAs are more effectively absorbed by dislocations. Physically, it is explained by the difference in dilatation volume and related stress-field topology between SIA and vacancy. The dislocation bias factor $\left(B_{\mathrm{d}}\right)$ is a quantity that defines the efficiency of absorption of these two defects. Following the GFR theory, the $B_{\mathrm{d}}$ was evaluated on the basis of experimentally measured void swelling on $\mathrm{Cu}$ and $\mathrm{FeCrNi}$ alloy upon $1 \mathrm{MeV}$ electron irradiation, which produces Frenkel pairs exclusively $[2,3]$. The dislocation bias was fitted from the rate theory model with experimental parameters to be in the range of 0.02-0.04. Heavy ion and neutron irradiation, resulting in cascade damage, also suggest the $B_{\mathrm{d}}$ to be of the order of a percent [4]. Importantly, the experimentally deduced value of the $B_{\mathrm{d}}$ was neither essentially dependent on the crystallographic structure of material nor its chemical composition, and swelling rate was typically reported to be of the order of $1 \% / \mathrm{dpa}$.

A computational assessment of $B_{\mathrm{d}}$ was done by Wolfer [5] who applied Ham's solution [6] and used isotropic elasticity theory to estimate the interaction of the point defects with a non-split edge dislocation in face centered 
cubic (fcc) $\mathrm{Cu} . B_{\mathrm{d}}$ was found to be 0.25 , that is, one order of magnitude higher than the expected value derived from the experimental observations. Estimations based on even simpler assumptions, accounting for the relaxation volume difference, also provide the values for $B_{\mathrm{d}}$ of the order of $0.1-0.3$ for different body centered cubic (bcc) and fcc metals [5]. In the above mentioned assessment, Wolfer mentioned that further refinement is necessary to account for the anisotropy in the long-range interaction and the particular atomic structure of the dislocation core and point defects for the short-range interaction. The effect of anisotropy of edge dislocation, as well as of the point defects, on dislocation bias calculations have recently been studied by Seif and Ghoniem [7] in different metals, where they found that the anisotropy plays different roles in fcc $\mathrm{Cu}$ and bcc Fe. Moreover, we have computed the $B_{\mathrm{d}}$ for an edge dislocation in fcc $\mathrm{Cu}$ by combining a finite element method with the interaction energy landscape obtained directly from atomistic calculations [8]. A discrepancy of $30 \%$ for $B_{\mathrm{d}}$ was found if the interaction energy map is taken from elasticity theory, and the interaction near the dislocation core also revealed strong deviations, sometimes even the sign of the interaction energy was inverted. Those results proved the usefulness of the atomistic simulations to assess fine details of defect-dislocation interactions which are not achievable using an elasticity theory framework only.

In this work, we investigate defect-dislocation interactions and perform calculations of $B_{\mathrm{d}}$ in bcc iron (Fe). In particular, we consider the $a_{0} / 2\langle 111\rangle\{110\}$ screw, $a_{0} / 2\langle 111\rangle\{110\}$ and $a_{0}\langle 100\rangle\{001\}$ edge dislocations. The screw dislocations are the primary defects in non-irradiated bcc metals and alloys including Fe-based steels [9], while under prolonged irradiation a high den- 
sity of dislocation loops is established [10,11]. The latter are of interstitial nature and two types may be present depending on the chemical composition of the Fe-based alloy and irradiation temperature. In pure Fe at $573 \mathrm{~K}$ and above, the $a_{0}\langle 100\rangle$ loops of square shape dominate, while at room temperature mostly $a_{0} / 2\langle 111\rangle$ loops are present $[12,10]$. In commercial Fe-Cr-based steels both types of loops are present in proportions depending on the $\mathrm{Cr}$ content $[13,14]$. Here, we provide a computational assessment of the dislocation bias factor for all three possible types of dislocations relevant for bcc Fe and its alloys and discuss the implication of the results.

\section{Theory and Methods}

The numerical method to obtain the dislocation bias has been explained in our previous work [8]. The basic idea is that the flux induced by the diffusion of point defects is described by Fick's law with a drift term, and it is solved under steady state by employing the dislocation - point defects interaction energies. The interaction energies are obtained both from atomistic calculations and from elastic calculations. For the aim of completeness, the theoretical equations to obtain the bias, the atomistic calculation details and the calculations of the elastic dislocation-PD interactions are briefly explained in the following sections.

\subsection{Bias factor}

The dislocation bias factor, sometimes referred to as net bias [15], is defined as:

$$
B_{\mathrm{d}}=\frac{Z_{\mathrm{SIA}}}{Z_{\mathrm{vac}}}-1
$$


where $Z_{\mathrm{SIA}}$ and $Z_{\text {vac }}$ are the dislocation capture efficiencies for the SIA and vacancy, respectively. The dislocation capture efficiency is a measure of enhanced absorption of a specific point defect by the dislocation due to its interaction with the point defect. The capture efficiency is determined by various factors such as the dislocation structure and resulting stress field, ambient temperature, dislocation density and others [16]. Previous works, dedicated to assessment of dislocation-mediated creep and swelling $[17,5,18]$, provide a framework to compute the capture efficiency $Z$. In the present work, we follow the numerical approach proposed by Wolfer [5], which is briefly explained below:

A flux of point defects (of a specific kind) approaching the dislocation core is influenced by both the concentration gradient and the gradient of the defect-dislocation interaction energy. It can be described by Fick's law with a drift term:

$$
J=-\nabla(D C)-\beta D C \nabla E
$$

where $D$ is the diffusion coefficient, $C$ is the concentration of point defects, and $\beta$ is $1 / k_{\mathrm{B}} T$ with $k_{\mathrm{B}}$ the Boltzmann constant and $T$ the temperature, $E$ is the defect-dislocation interaction energy and the second term on the right hand side is the drift term.

A convenient reformulation is used:

$$
J=-e^{-\beta E(r, \theta)} \nabla \Psi
$$

where $\Psi=D C e^{\beta E(r, \theta)}$ is referred to as the diffusion potential function.

Applying the steady state condition $\nabla \cdot J=0$ in Eq.3: 


$$
\nabla \cdot\left(-e^{-\beta E(r, \theta)} \nabla \Psi\right)=0 .
$$

Given the interaction energy $E(r, \theta)$ and boundary conditions, $\Psi$ is numerically solved using FEM. In this work, atomistically calculated interaction energies and elastic interaction models are implemented accordingly in different calculations. Following the conventional approach [5], it is assumed that a point defect is absorbed by the dislocation once it crosses into the core region. Hence, the boundary condition at the dislocation core $r=r_{0}$, is $\Psi_{r_{0}}=0$. At the external boundary, limited by the dislocation capture range, $r=R$, the defect concentration $C(r, \theta)$ is a constant. Naturally, the dislocation capture range corresponds to a distance at which the dislocation-defect interaction is negligible. Hence, $\Psi_{R}=\bar{D} \bar{C}$ is a non-zero constant.

The total current of defects absorbed by the dislocation is then evaluated as:

$$
J_{\text {tot }}=r_{0} \int_{0}^{2 \pi} J_{r}\left(r_{0}, \theta\right) d \theta
$$

where $r_{0}$ is the dislocation core radius vector pointing to the core center and $J_{r}\left(r_{0}, \theta\right)$ is the current to the core.

The dislocation capture efficiency $Z$ in this case is defined as the ratio of the fluxes for a specific point defect, computed with and without taking into account the dislocation-defect interaction, that is $Z=\frac{J}{J_{0}}$, where $J_{0}$ is the flux excluding the dislocation-defect interaction. The dislocation gliding plane is mounted with a mesh, on each mesh point the flux is obtained by FEM implementing the interaction energy on each specific mesh site. The total flux around the dislocation core is then integrated to obtain the capture efficiency. The latter is used to compute the bias factor. 
A similar numerical method has recently been used to calculate the effect of anisotropy, SIA orientation, and one-dimensional migration mechanism on the bias of edge dislocations in bcc Fe and fcc $\mathrm{Cu}$ [7]. Different elastic interaction models have been employed in their work. In the present work, in addition to the anisotropic elastic interaction model, a number of atomistic interaction energy maps are calculated from molecular static calculations and we implement them in the FEM numerical method to obtain the dislocation bias on different types and configurations of dislocation in bcc Fe. Details of the atomistic calculation settings are found in the following section.

\subsection{Atomistic calculations}

To obtain the atomistic information about the defect-dislocation interaction, a model treating a periodic array of dislocations by Osetky and Bacon [19] was applied. In the case of edge dislocations, two half crystals, where one has an extra plane of atoms, are strained to have different lattice parameters in the direction of the Burgers vector. For the screw dislocation, a rigid shift in the periodic boundary conditions perpendicular to the dislocation line was applied, as suggested by Rodney [20]. In order to model an infinite straight dislocation, periodic boundary conditions were applied in the direction of the Burgers vector and in the direction of the dislocation line, while fixed boundary condition was applied in the direction that is normal to the glide plane. Typical dimensions of the simulation cells were about $40 \times 40 \times 4 \mathrm{~nm}$, where $4 \mathrm{~nm}$ is the section along the dislocation line. These dimensions are enough to avoid defect-defect self-interaction via the periodic boundary along the dislocation line, as well as dislocation-dislocation image interactions which could possibly affect the equilibrium core structure. The 
total number of atoms in these simulation cells system is about $5 \cdot 10^{5}$, and the dislocation density is of the order of $5 \cdot 10^{14} \mathrm{~m}^{-2}$.

A vacancy is created by removing one atom from the lattice. A SIA is inserted as a dumbbell containing two atoms aligned along $\{110\}$ directions and placed at a distance of $0.4 a_{0}$ from each other, centered on a lattice site. A combination of conjugate gradient and quasi static relaxation in the microcanonical ensemble was applied to fully relax the crystal to obtain its total energy. The formation energies with and without the dislocation are calculated and the interaction energy is defined as the difference in formation energy with and without the dislocation. The interaction energy with respect to the dislocation core-defect position provides the interaction energy map for a particular dislocation-defect combination. Note that for a SIA, being a $\langle 110\rangle$ dumbbell in bcc Fe [21, 22], the particular orientations of the dumbbells also play a role, meaning that all non-equivalent configurations must be assessed.

Most of the calculations were performed using the embedded atom method (EAM) potential derived by Dudarev and Derlet [23]. The potential was developed to account for the specific properties of SIAs and ensures a correct stability of the $\langle 110\rangle$ dumbbell configuration over the $\langle 111\rangle$ crowdion, which is of fundamental importance in the present investigation. Despite the fact that the potential was fitted to a number of important properties of bcc Fe, the core of the screw dislocation relaxes to a degenerate three-fold structure. To validate the effect of the core structure, we also employed the EAM potential developed by Mendelev et al. [24], which correctly reproduces both properties of point defects and screw dislocation core structure [25]. The 
results show that the $Z$ and $B_{\mathrm{d}}$ values computed for the screw dislocation by both potentials are qualitatively in agreement. We therefore choose to use the potential of Dudarev and Derlet, which has one obvious advantage over the Mendelev potential, namely the shorter cut-off range. This cut-off range is crucial for the present study due to the significant computational resources required to assess the interaction energy landscapes in crystals containing $5 \cdot 10^{5}$ atoms while considering six variants of the SIA and three types of dislocations.

\subsection{Analytical screw dislocation-defect interaction}

In this work we compare the analytical dislocation-defect interactions derived from elasticity theory with the results from atomistic calculations. Given that the Zener anisotropy factor of bcc Fe is 2.3, it is not appropriate to treat it as an isotropic bulk. Therefore, only the anisotropic dislocation model is used in this work to compare with the atomistically calculated dislocation. Our treatment of the anisotropic interaction between edge dislocation and PDs is explained in [8]. All elastic constants $C_{i j}$ used in the elastic interaction models are calculated from molecular static calculations using the same EAM potential as we used in the atomistic interaction energy calculations. In this section the anisotropic interaction model is explained for the interaction of the point defects with a screw dislocation, as briefly described below.

Within the framework of linear elasticity theory, the interaction energy between the point defect and a dislocation separated by a distance $r$ is given by

$$
E(r)=-\epsilon_{i j} P_{i j}
$$


where $\epsilon$ is the strain field of the dislocation and $P_{i j}$ are components of the dipole force tensor $\mathrm{P}$.

The anisotropic strain field of a screw dislocation was calculated from the anisotropic stress field given by[26]. A dipole force tensor describes the influence that a point defect has on its neighbours. It is calculated following the standard method by obtaining the Kanzaki force [27, 22, 28]

$$
P_{i j}=\sum_{k=1}^{N} R_{i}^{(k)} F_{j}^{(k)},
$$

where the summation is over $N$ neighbours of the defect, $i$ and $j$ are the directions, $R$ is the $i$ th component of the vector joining atom $k$ and the central atom, and $F$ is the Kanzaki force.

The Kanzaki force for our EAM potential is directly calculated from molecular statics following the detailed descriptions in [27, 28]. The dipole tensor of the vacancy and of the six different configurations of $\langle 110\rangle$ dumbbells are calculated and then used individually in the interaction calculation of Eq.6. The interaction energy of a screw dislocation with a SIA is presented by the average interaction energies of the screw dislocation with the six dumbbell orientations.

\section{Results and discussions}

\subsection{Dislocation core radius}

A standard way to define the dislocation core is to assign a cylinder with a radius (henceforth core radius) within which a spontaneous absorption of a point defect is expected. The core radius is therefore defined by the strength 
and topology of the dislocation-defect interaction. In the analytical expression, the capture efficiency is predicted to be core radius dependent [29]. In our case, the interaction energy is obtained from atomistic calculations, therefore the core radii must be chosen carefully. To begin, we analysed the sensitivity of the capture efficiency with regard to the dislocation core radius, assuming that the core is represented by a cylinder. Fig. 1 shows $Z_{\text {SIA }}$ and $Z_{\text {vac }}$ calculated using the energy landscape from isotropic elasticity theory and atomistic simulations, for the $\langle 100\rangle$ edge dislocation as a function of $r_{0}$. The integration was performed by taking $873 \mathrm{~K}$ as ambient temperature and assigning the dislocation density to $10^{14} \mathrm{~m}^{-2}$. As one sees, the capture efficiency $Z$ values are strongly dependent on the choice of $r_{0}$, especially in the case of the SIA. When $r_{0}$ is larger than around $24 \AA$, the interaction energy becomes too weak to overcome the influence of the thermal diffusion. To assign some physical meaning to the dislocation core radius, we used an interaction energy gradient threshold [7], resulting in different dislocation radii for different species. Inside the radius all defects of that species are absorbed by the dislocation. The dislocation core radius is thus determined as where the gradient of the interaction energy, scaled by the Burgers vector, is comparable to the thermal energy:

$$
b \nabla|E|=k_{\mathrm{B}} T .
$$

This criterion is applied to obtain the capture radii on each individual combination of defects and dislocation, in the atomistic interaction maps and in the elastic interaction maps. The average interaction gradient on the radius is used. However, in the here used temperature range, the $\nabla|E|$ difference is not significant, and we therefore use the radii corresponding to 
$T=873 \mathrm{~K}$ for all temperatures. This results in core radii as shown in Tab.1.

\subsection{Bias factor for edge dislocations}

While in most of the bcc metals SIAs typically occupy $\langle 111\rangle$ crowdion configurations [30], a $\langle 110\rangle$ dumbbell is the most energetically favourable configuration in bcc Fe due to its magnetism [22]. Occupying such a configuration, the SIA performs 3D migration by translation-rotation jumps [21, 31]. While the SIA migrates towards the dislocation core it will undergo constant change of the particular orientation of its $\langle 110\rangle$ axis. It is therefore important to assess the interaction energy landscape for all possible variants of SIA-dislocation orientations. For the $\langle 100\rangle$ dislocation, the high symmetry results in three orientations of the $\langle 110\rangle$ dumbbells. However, the $1 / 2\langle 111\rangle$ dislocations retain a high assymmetry. This has been taken into account in our calculations. In order to represent the interaction energy for the constant change of the particular orientation of a $\langle 110\rangle$ dumbbell, the average interaction energy on each lattice site is calculated from each individual interaction energy map of a given orientation. The near core interaction fields are plotted for both types of edge dislocation, including the atomistic calculation and elastic anisotropic models. The profile of anisotropic interactions of the $\langle 100\rangle$ type with the point defects, shown in Fig.2 as C and D, resemble the atomistic interactions, shown in Fig.2 as A and B, very well, with weaker interactions on each individual site. On the other hand, the atomistic interaction profiles for the $1 / 2\langle 111\rangle$ type, shown in Fig.3 as A and B, do not have high similarity to either of the analytical interaction models.

The bias calculated from those interaction maps are shown in Fig.4 under different temperatures with a fixed dislocation density of $10^{13} \mathrm{~m}^{-2}$. Compar- 
ing the bias obtained from the two types of dislocation using the atomistically calculated interaction maps, a larger bias factor is observed for the $1 / 2\langle 111\rangle$ type than for the $\langle 100\rangle$ type. However, the opposite result is obtained from the elastic anisotropic models. That is, the bias factors for the $\langle 100\rangle$ type are larger than those for the $1 / 2\langle 111\rangle$ type. This follows the argument based on the conventional estimation of $B_{\mathrm{d}}$, that the larger the Burgers vector, the higher the bias factor should be [32]. However, such a view neglects particular details of the defect-dislocation interaction in the vicinity of the dislocation core, and the argument was not in agreement with the experiment carried out by Katoh [33] under neutron irradiation. By comparing Fig.2 A, B and Fig.3 A, B, the attractive regions (negative interaction energy region) in the SIA-dislocation interaction maps, which are marked as subplot $\mathrm{B}$ in both figures, are much more significant than the attractive regions in the vacancy-dislocation interaction maps, which are marked as subplot A. This difference ensures a stronger sink capture efficiency for SIAs than for vacancies in both cases. However, the repulsive region of the SIA-dislocation interaction is more pronounced for the $\langle 110\rangle$ (Fig.2) compared to that for the $1 / 2\langle 111\rangle$ (Fig.3) dislocations. The large repulsive region screens out SIAs approaching from that side, resulting in a relatively small dislocation bias for the $\langle 100\rangle$ type. As the sink capture efficiency values show in Fig.6, it is the diffusion of SIAs to the dislocation that dominates the difference in the dislocation bias factors for the two edge dislocations. It is worth mentioning that even though the atomistic- and anisotropic elastic $B_{\mathrm{d}}$ values for the $1 / 2\langle 111\rangle$ are similar, this is coincidental considering the significant differences in the individual capture efficiencies. 


\subsection{Bias factor for the screw dislocation}

The screw dislocation is of primary interest since it is the basic extended lattice defect in non-irradiated bcc metals. The interaction energies obtained from the atomistic calculations and the anisotropic analytical models are shown in Fig.5. Three-fold interaction symmetries are obtained. The main difference between the atomistic calculated interaction energy and the analytical interaction energy is in the core region. For both vacancy and SIA cases, the interactions obtained from the atomistic calculations are much stronger and longer in range than the analytical ones. Comparing Fig.5 with Fig.2 and Fig.3, the interaction energy of the screw dislocation is much weaker than that of the edge dislocations. The capture efficiencies have been computed for the screw dislocation as a function of temperature and dislocation density, as shown in Fig.6 and Fig.7. The results show that $Z_{\text {vac }}$ are larger than $Z_{\text {SIA }}$ in all conditions tested. That is to say, the $B_{\mathrm{d}}$ of a screw dislocation calculated using atomistic interactions are all negative. In the SIA case the reason for this is that the flux approaching the core is partly repelled by the strong compressive fields around it, while in the vacancy case there are no such strong repelling fields and the attractive interaction energies between the two cases are comparable. This results in a larger net flux into the core for the vacancy than for the SIA. The same pattern of the interaction energy and the same trend for the dislocation bias are observed using the Mendelev potential, with even stronger repulsion zones and therefore even larger negative capture efficiency and bias values. The negative bias implies that more vacancies than SIAs are absorbed in the dislocations. The supersaturation of the SIAs left in the bulk should help to build up edge dislocation loops or 
help the growth of existing loops.

Furthermore, the $B_{\mathrm{d}}$ calculated using the analytical interactions are zero within the precision limits. Given the fact that there is no displacements on the plane perpendicular to the dislocation line in a screw dislocation, only a very weak stress field from the anisotropy on this plane exert an influence on any point defects. It is hence reasonable for the analytical interactions not to have any impact on the preferential absorption rate. On the other hand, the linear elastic description breaks down in the core region and hence the atomistic interaction is more realistic. The negative bias implies that a screw dislocation preferentially absorbs vacancies. Nonetheless, in a real irradiated material, the screw dislocations and edge dislocations usually coexist [9]. More discussions are presented in the next subsection.

\subsection{Comparison of screw- and edge dislocations}

Fig.6 and Fig.7 show capture efficiencies $Z$ as a function of temperature and dislocation density, respectively. Fig.6 shows data produced by taking the dislocation density to be $10^{13} \mathrm{~m}^{-2}$, while the data in Fig. 7 corresponds to $773 \mathrm{~K}$. The results reveal that $Z$ grows as dislocation density increases and temperature decreases. Such trends are consistent with common expectations, since temperature effectively 'weakens' the interaction energy gradient, while the dislocation density controls the available sink volume. The same trends were found in our recent study dedicated to the calculation of $B_{\mathrm{d}}$ in fcc $\mathrm{Cu}[8]$.

For the edge dislocations, $Z_{\text {SIA }}$ are larger than $Z_{\text {vac }}$, while it is the opposite for the screw dislocation: $Z_{\text {SIA }}$ are smaller than $Z_{\mathrm{vac}}$. As we mentioned before, the coexistence of edge- and screw dislocations contribute to macroscopic 
effects such as swelling. Therefore it is more reasonable to look at the total effect of the $Z$ values. In order to estimate the joined influence from them, we define a total dislocation bias. It is assumed that the densities of the three different types of dislocations, namely $1 / 2\langle 111\rangle$ screw, $1 / 2\langle 111\rangle$ edge and $\langle 100\rangle$ edge, are $a, b$ and $c$ respectively, and that the point defects can be absorbed by any of them, then the total bias is defined as

$$
B_{\mathrm{d}}=\frac{a Z_{\mathrm{SIA}}^{S D}+b Z_{\mathrm{SIA}}^{<111>}+c Z_{\mathrm{SIA}}^{<100>}}{a Z_{\mathrm{vac}}^{S D}+b Z_{\mathrm{vac}}^{<111>}+c Z_{\mathrm{vac}}^{<100>}}-1
$$

where SD represents the screw dislocation, $\langle 111\rangle$ is the $1 / 2\langle 111\rangle$ type edge dislocation and $\langle 100\rangle$ represents the $\langle 100\rangle$ type edge dislocation.

The fact that $Z_{\mathrm{vac}}>Z_{\mathrm{SIA}}$ in the screw dislocation case counteracts the bias from the edge dislocation. This balancing of the total dislocation bias could indicate a contributing mechanism for the swelling incubation in bcc metals, as explained briefly below.

Following the analysis of bias factors for different types of dislocations, we suggest that the microstructure-driven interplay balancing the sinks of point defects could be the feature defining the onset of stable (i.e. steady-state) void swelling in bcc metals. The above mentioned interplay with progressing irradiation is expressed qualitatively in the following. The unirradiated material contains predominantly screw dislocations. During irradiation, the edge dislocation population will be built up in the form of dislocation loops, whose properties will approach that of straight edge dislocations when large enough. With increasing irradiation dose, the total length of edge dislocations will keep increasing. Thus, at a certain dose, the positive bias of edge dislocations will outbalance the negative bias of screw dislocations and a bias 
driven swelling sets in, according to Eq.9. Further investigation is needed to put this scenario on a firm quantitative ground, specifically the bias of $2 \mathrm{D}$ dislocation loops would need to be quantified, and the irradiation-induced microstructure evolution would need to be explicitly modelled. However, it has been previously noted that the transition from an incubation region to swelling is linked to a qualitative change in the microstructure as well as the absolute swelling correlates with the density of radiation-induced dislocation loops [34, 35, 33], and the above suggested scenario is well in line with those findings.

\section{Conclusions}

In this work we have performed a computational assessments of capture efficiencies and bias factors for screw- and edge dislocations in bcc Fe. $B_{\mathrm{d}}$ was computed by combining information from atomistic simulations and the finite element calculation approach. Atomistic calculations were used to obtain the interaction energy landscape for SIA/vacancy-dislocation interactions.

An unexpected result was obtained for the $1 / 2\langle 111\rangle$ edge dislocation, whose $B_{\mathrm{d}}$ was found to be higher than that for the $\langle 100\rangle$ edge dislocation. At first glance this result contradicts the general perception that $B_{\mathrm{d}}$ is proportional to the dislocation stress-field and hence proportional to the absolute value of the Burgers vector. The atomistic calculations of the interaction landscapes show that the near core region is essentially different for the two edge dislocations. The stronger and broader repulsive region in the $\langle 100\rangle$ edge-SIA interaction map plays an important role in screening out SIAs approaching from that side and thus explains the unexpectedly low $B_{\mathrm{d}}$ for the 
$\langle 100\rangle$ edge dislocation.

For the screw dislocation, negative bias values $\left(Z_{\mathrm{vac}}>Z_{\mathrm{SIA}}\right)$ were obtained using atomistic interaction energies and negligible bias was obtained using the analytical models. These results can be readily understood by comparing the interaction energy landscapes that the atomistic and analytical models predict. For the atomistic case, three repulsion zones drive away SIAs but not vacancies. For the analytical case, the interaction energies are almost negligible.

The effect of dislocation density on $Z$ was assessed for the temperature range $623-823 \mathrm{~K}$, which is typical for the application of Fe-based ferric steels (e.g. high $\mathrm{Cr}$ steels) in the nuclear industry where the swelling phenomenon is a practical issue. It is found that while a temperature increase leads to the reduction of $Z$, the increase of dislocation density causes an increase of $Z$. A combination of the $Z$ values from edge- and screw dislocations is used to assess the joint influence. A possible supplementary mechanisms of the long incubation time in the bcc material has been suggested from the point of the view of the joint dislocation bias.

\section{Acknowledgements}

This work is supported by the national project on Generation IV reactor research and development (GENIUS) in Sweden, by the Göran Gustafsson Stiftelse and by the European Atomic Energy Community's (Euratom) Seventh Framework Programme FP7/2007-2013 under grant agreement No.604862 (MatISSE project). This work contributes to the Joint Program on Nuclear Materials (JPNM) of the European Energy Research Alliance (EERA). The 
Swedish National Infrastructure for Computing (SNIC) sources are used for part of this work.

[1] G. Greenwood, A. Foreman, D. Rimmer, J. Nucl. Mater. 1 (1959) 305324.

[2] S. Golubov, B. Singh, H. Trinkaus, Philos. Mag. A 81 (2001) 2533-2552.

[3] G. Walters, J. Nucl. Mater. 136 (1985) 263-279.

[4] F. Garner, M. Toloczko, B. Sencer, J. Nucl. Mater. 276 (2000) 123-142.

[5] W. Wolfer, J. Comput. Aided Mater. Des. 14 (2007) 403-417.

[6] F. S. Ham, J. Appl. Phys. 30 (1959) 915-926.

[7] D. Seif, N. Ghoniem, J. Nucl. Mater. 442 (2013) S633 - S638.

[8] Z. Chang, P. Olsson, D. Terentyev, N. Sandberg, J. Nucl. Mater. 441 (2013) 357-363.

[9] J. Nagakawa, A. Sato, M. Meshii, Philos. Mag. 32 (1975) 1107-1128.

[10] S. J. Zinkle, B. N. Singh, J. Nucl. Mater. 351 (2006) 269-284.

[11] N. Yoshida, M. Kiritani, F. Eiichi Fujita, J. Phys. Soc. Jpn. 39 (1975) $170-179$.

[12] M. Jenkins, C. English, B. Eyre, Philos. Mag. A 38 (1978) 97-114.

[13] S. Porollo, A. Dvoriashin, A. Vorobyev, Y. V. Konobeev, J. Nucl. Mater. 256 (1998) 247-253. 
424

425

426

427

428

429

430

431

432

433

434

435

436

437

438

439

440

441

442

[14] D. Gelles, J. Nucl. Mater. 108 (1982) 515-526.

[15] W. Wolfer, R. Konings, Comprehensive nuclear materials. Oxford: Elsevier (2012) 1-45.

[16] L. Mansur, A. Brailsford, W. Wolfer, J. Nucl. Mater. 105 (1982) 36-38.

[17] P. Heald, Philos. Mag. 31 (1975) 551-558.

[18] W. Wolfer, M. Ashkin, J. Appl. Phys. 47 (1976) 791-800.

[19] Y. N. Osetsky, D. J. Bacon, Modell. Simul. Mater. Sci. Eng. 11 (2003) 427.

[20] D. Rodney, Acta Mater. 52 (2004) 607-614.

[21] R. Johnson, Phys. Rev. 134 (1964) A1329.

[22] C. Domain, C. Becquart, Phys. Rev. B 65 (2001) 024103.

[23] S. Dudarev, P. Derlet, J. Phys.: Condens. Matter 17 (2005) 7097.

[24] M. Mendelev, S. Han, D. Srolovitz, G. Ackland, D. Sun, M. Asta, Philos. Mag. 83 (2003) 3977-3994.

[25] J. Chaussidon, M. Fivel, D. Rodney, Acta Mater. 54 (2006) 3407-3416.

[26] Y. Chou, T. Mitchell, J. Appl. Phys. 38 (1967) 1535-1540.

[27] G. Simonelli, R. Pasianot, E. Savino, Phys. Rev. B 50 (1994) 727.

[28] E. Hayward, C. Deo, B. P. Uberuaga, C. N. Tomé, Philos. Mag. 92 (2012) 2759-2778. 
443

444

445

446

447

448

449

450

451

452

453

454

455

[29] L. K. Mansur, Nucl. Technol. 40 (1978) 5-34.

[30] D. Nguyen-Manh, A. Horsfield, S. Dudarev, Phys. Rev. B 73 (2006) 020101.

[31] F. Willaime, C. Fu, M. Marinica, J. Dalla Torre, Nucl. Instr. Meth. Phys. Res. B 228 (2005) 92-99.

[32] R. Bullough, M. Wood, E. Little, ASTM special technical publication (1981) 593-609.

[33] Y. Katoh, A. Kohyama, D. S. Gelles, J. Nucl. Mater. 225 (1995) 154162.

[34] E. Little, R. Bullough, M. Wood, Proceedings of the Royal Society of London. Series A, Mathematical and Physical Sciences (1980) 565-579.

[35] Y. V. Konobeev, A. Dvoriashin, S. Porollo, F. A. Garner, J. Nucl. Mater. 355 (2006) 124-130. 
Table 1: Dislocation core radii that are used in this work.

\begin{tabular}{ccccc} 
& \multicolumn{2}{c}{ Atomistic } & \multicolumn{2}{c}{ Elastic model } \\
\hline & vacancy & SIA & vacancy & SIA \\
Edge $\langle 100\rangle$ & $9 \AA$ & $12.5 \AA$ & $5 \AA$ & $8 \AA$ \\
Edge $1 / 2\langle 111\rangle$ & $8 \AA$ & $13 \AA$ & $5 \AA$ & $8 \AA$ \\
Screw $1 / 2\langle 111\rangle$ & $6.5 \AA$ & $8 \AA$ & $5 \AA$ & $7 \AA$ \\
\hline
\end{tabular}




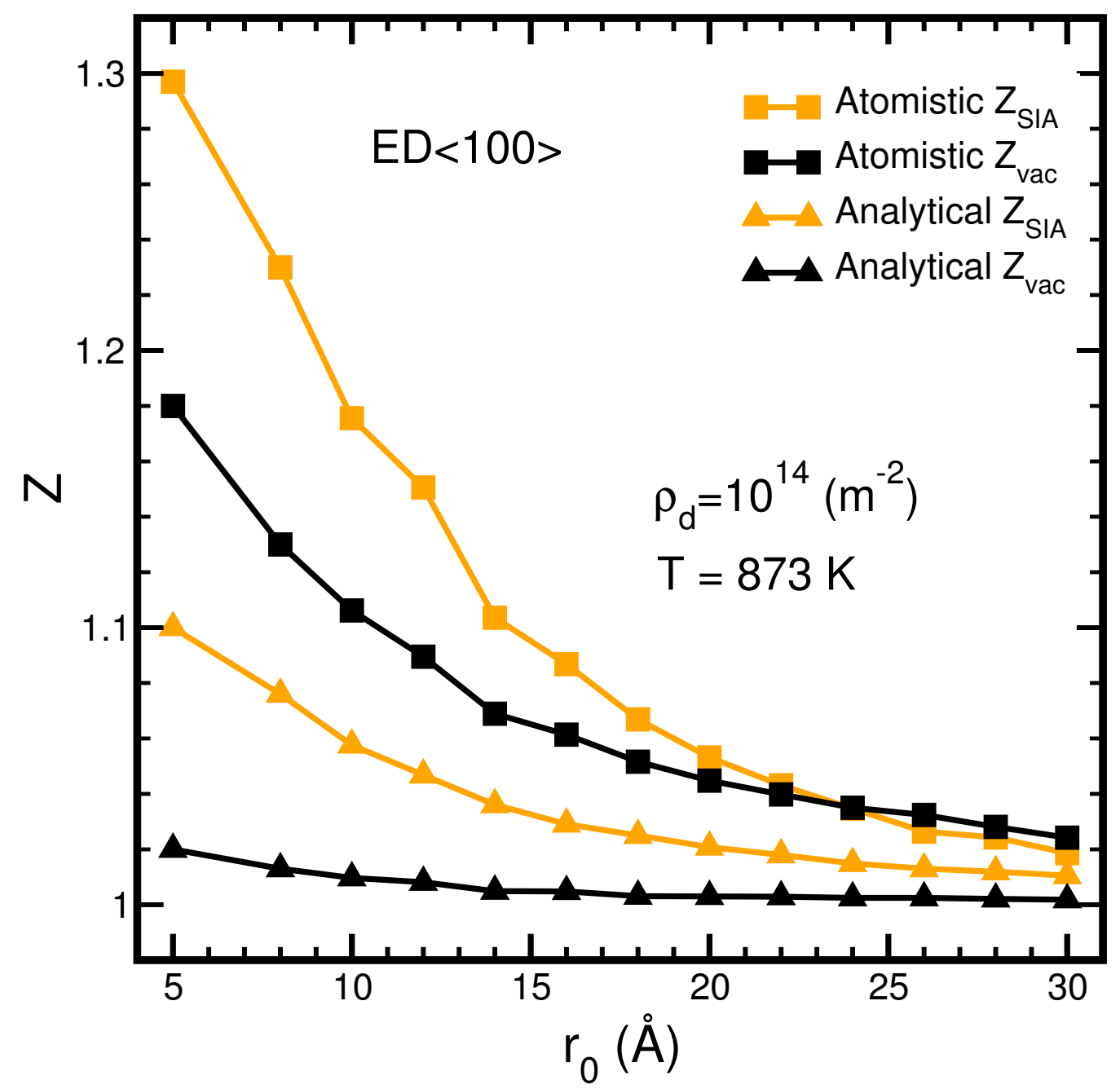

Figure 1: The effect of the dislocation core radii on the capture efficiencies of edge dislocation $\langle 100\rangle\{001\}$ type. Atomistic and analytical represent the atomistic and analytical interaction energy. 


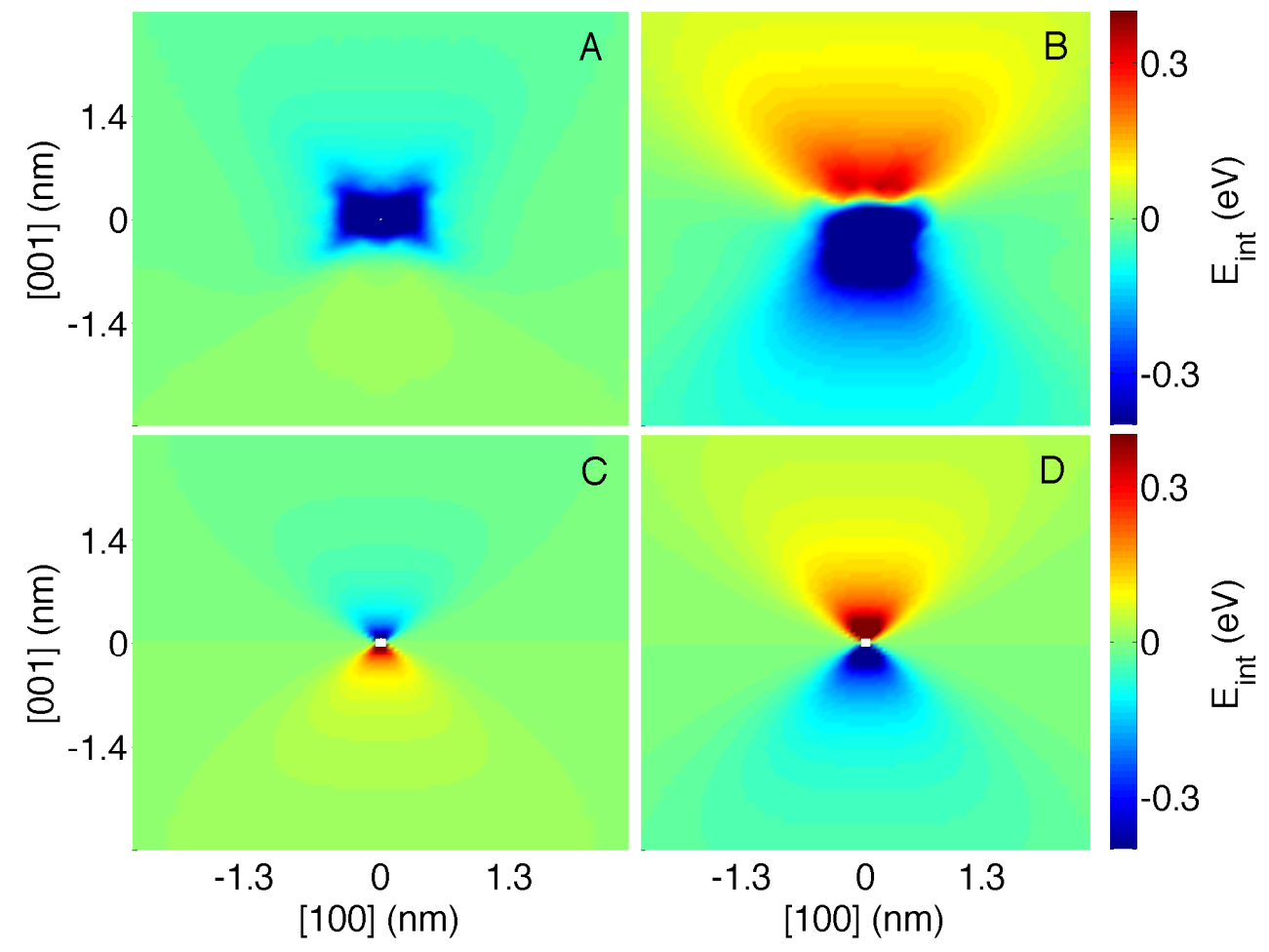

Figure 2: Interaction energies with the edge dislocation of $\langle 100\rangle\{001\}$ type. A. the atomistically calculated interaction between the dislocation and a vacancy; B. the atomistically calculated average interaction between the dislocation and all the nonequivalent $\langle 110\rangle$ SIAs; C. the anisotropic interaction energies between the dislocation and a vacancy; D. the anisotropic interaction energies between the dislocation and a $\langle 110\rangle$ SIA. 


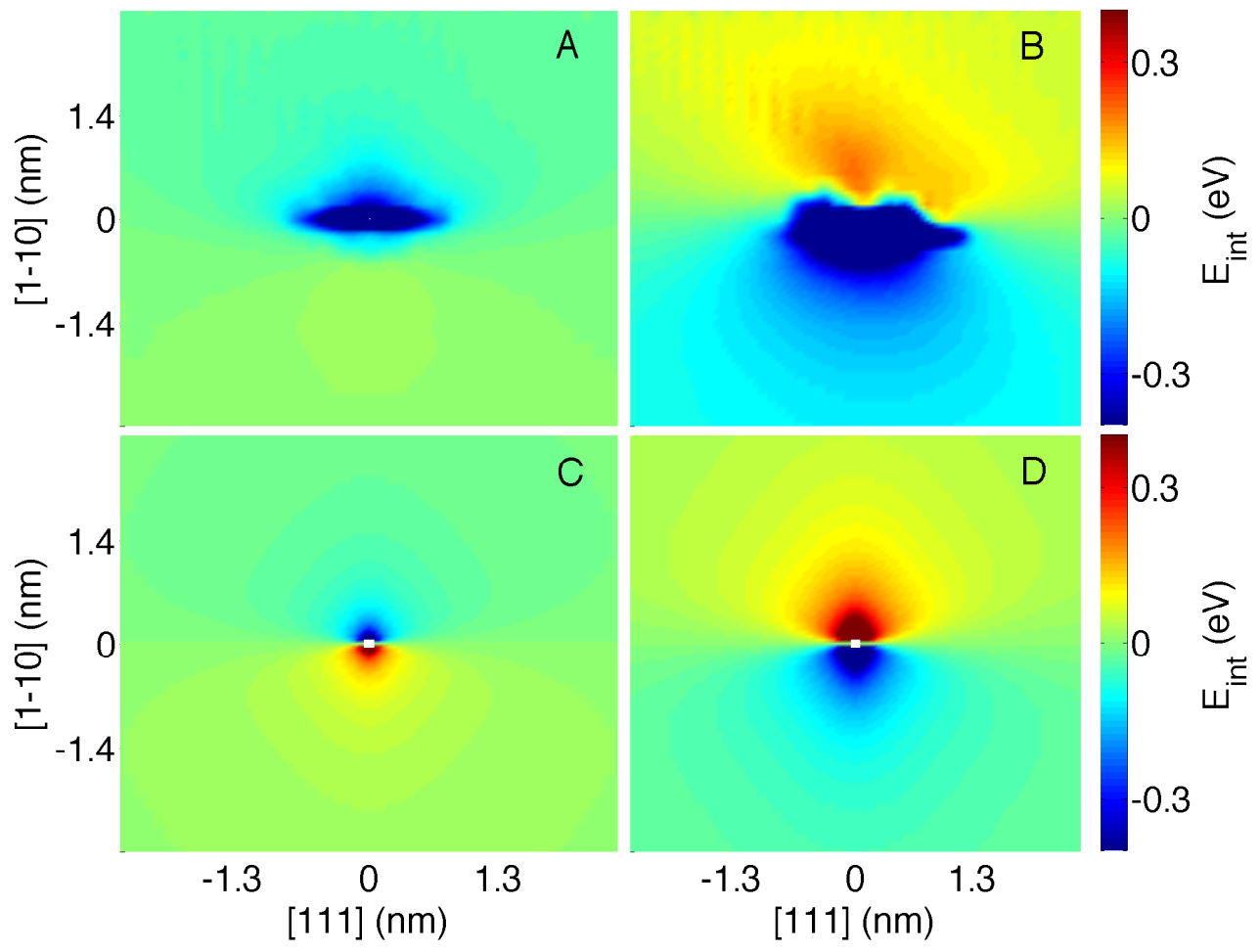

Figure 3: Interaction energies with the edge dislocation of $1 / 2\langle 111\rangle\{110\}$ type. A. the atomistically calculated interaction between the dislocation and a vacancy; B. the atomistically calculated average interaction between the dislocation and all the nonequivalent $\langle 110\rangle$ SIAs; C. the anisotropic interaction energies between the dislocation and a vacancy; D. the anisotropic interaction energies between the dislocation and a $\langle 110\rangle$ SIA. 


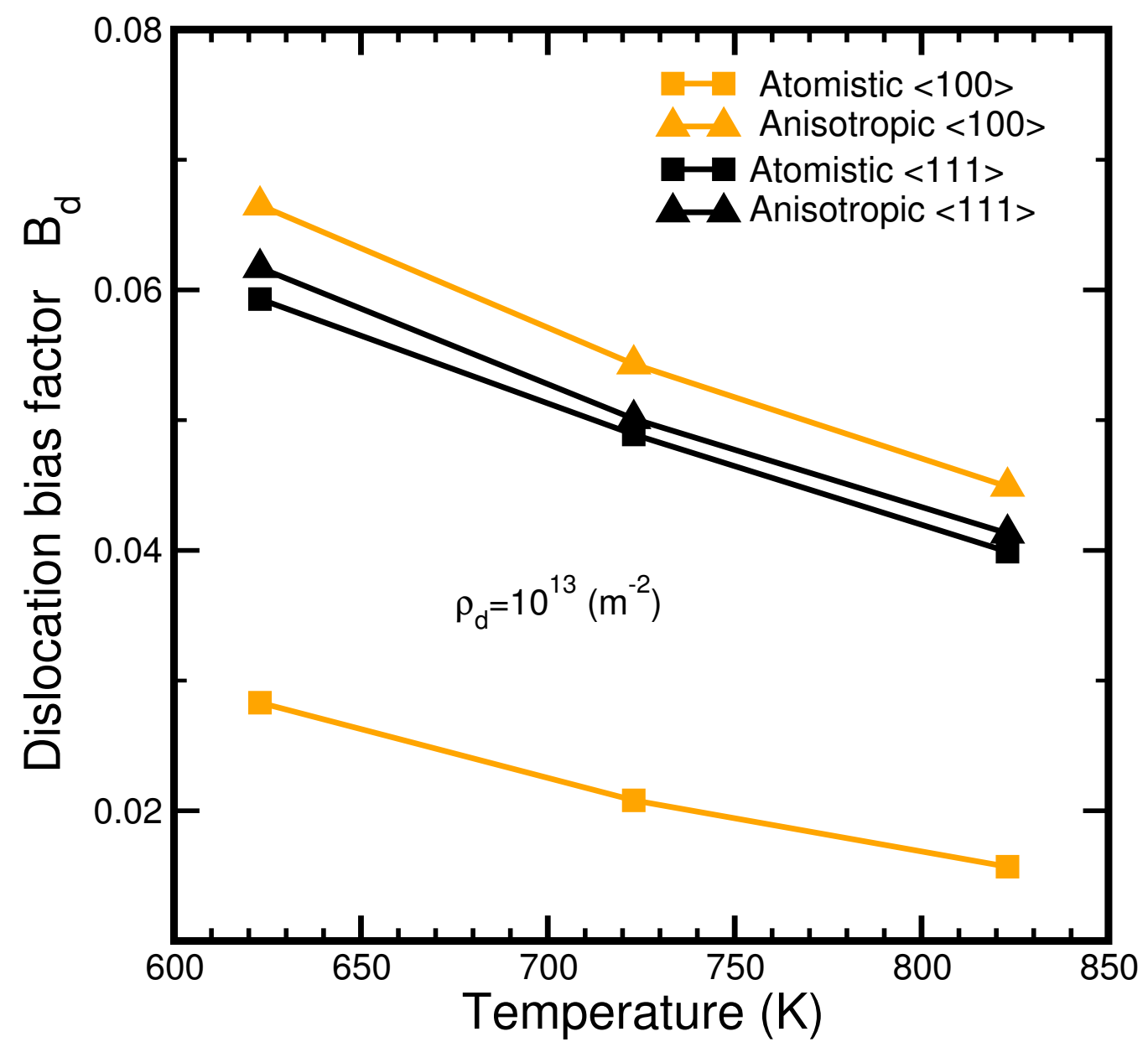

Figure 4: The dislocation bias of both types edge dislocations. $\langle 100\rangle$ and $\langle 111\rangle$ represent $\langle 100\rangle\{001\}$ type and $1 / 2\langle 111\rangle\{110\}$ type, respectively. 


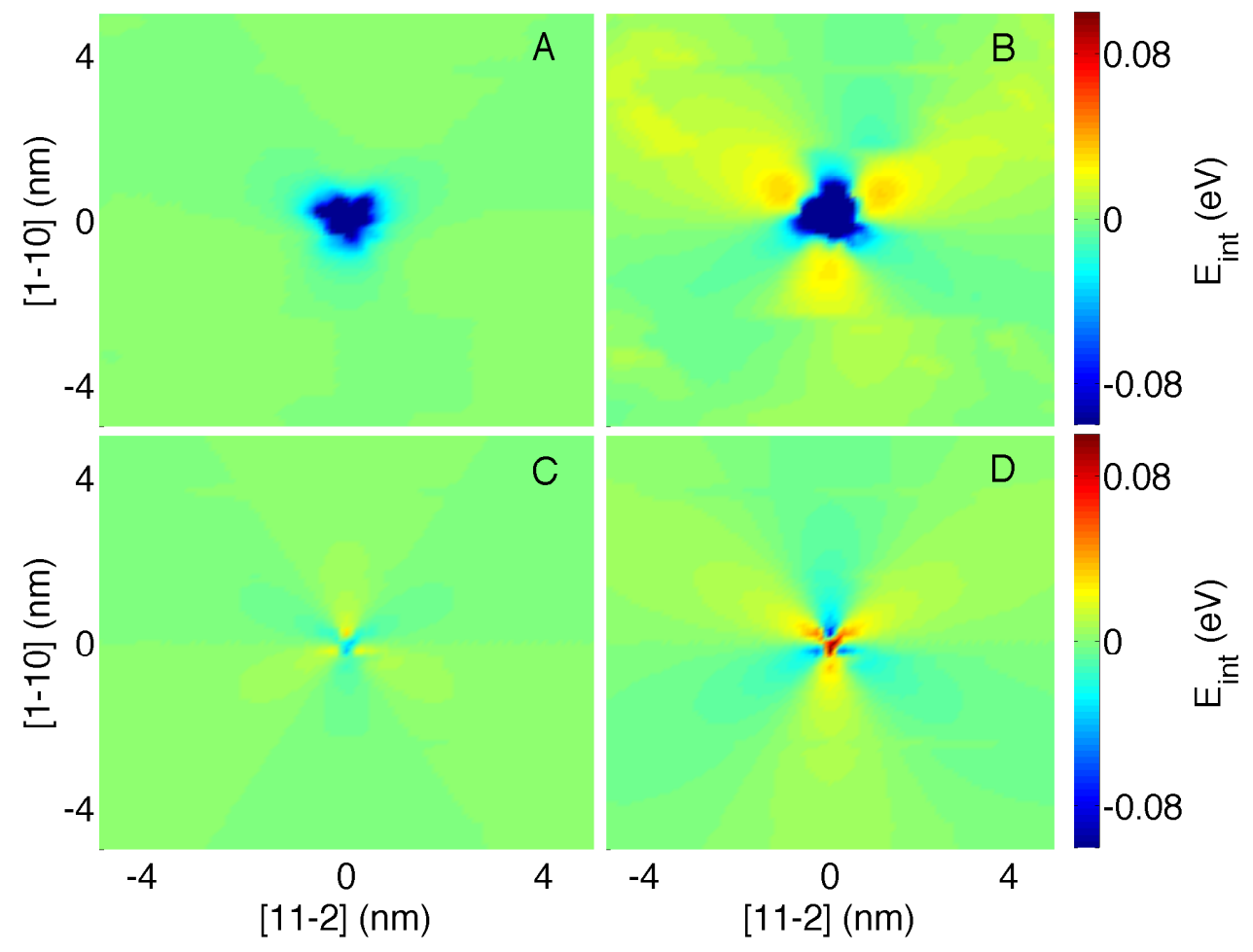

Figure 5: Interaction energies with the screw dislocation of $1 / 2\langle 111\rangle\{110\}$ type. A. the atomistically calculated interaction between the dislocation and a vacancy; B. the atomistically calculated average interaction between the dislocation and all the nonequivalent $\langle 110\rangle$ SIAs; C. the anisotropic interaction energies between the dislocation and a vacancy; D. the anisotropic interaction energies between the dislocation and a $\langle 110\rangle$ SIA. 


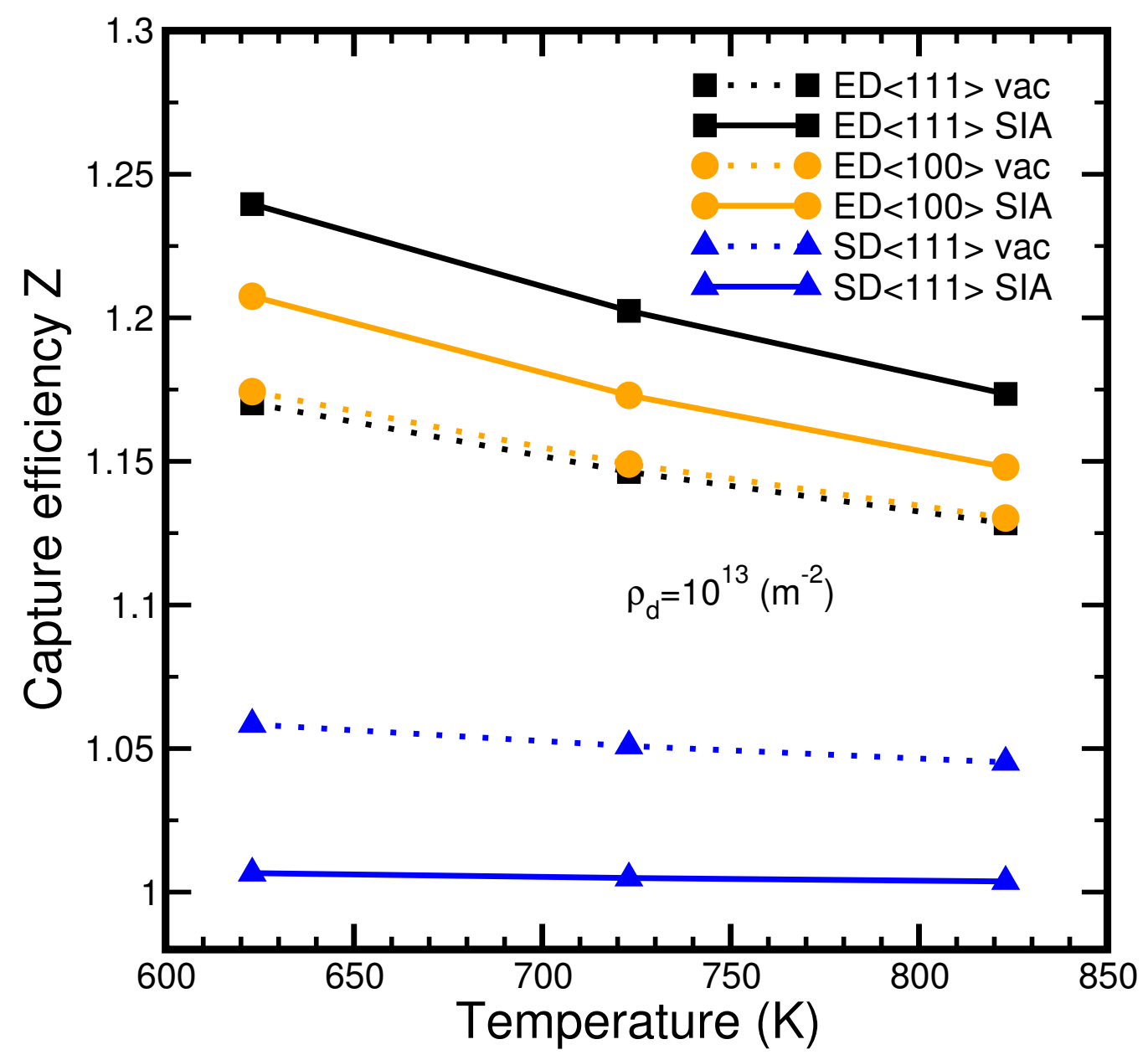

Figure 6: Comparison of capture efficiencies of the three kinds of dislocations as a function of temperature. SD and ED represent screw dislocation and edge dislocation respectively. 


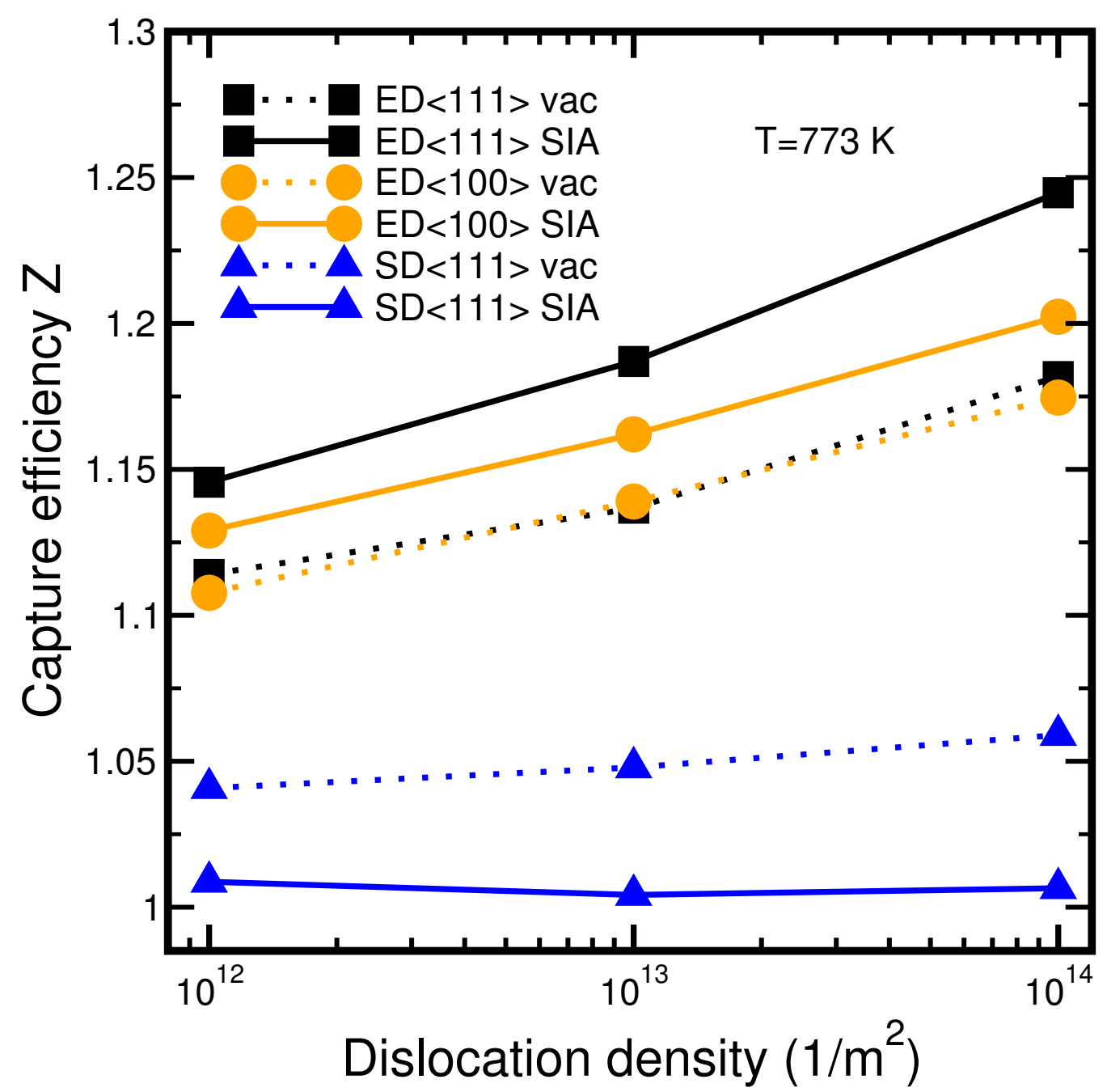

Figure 7: Comparison of the capture efficiencies of the three kinds of dislocations as a function of temperature. SD and ED represent screw dislocation and edge dislocation respectively. 